\title{
Nuclear medicine in psychiatry
}

Piotr Lass, Jarosław Sławek

Department of Nuclear Medicine, Medical University, Gdańsk, Poland Department of Neurological Nursing, Medical University, Gdańsk, Poland

[Received 28 IX 2007; Accepted 19 X 2007]

\section{Introduction}

From the second half of the twentieth century, advances in biological and psychopharmacological research seemed to promise a swift discovery of an organic basis of psychiatric diseases. Today we know that biological research in psychiatry is not a success story. This may be due to the complicated character of psychiatric disorders, where it is difficult to establish a unified model of the disease and thus in many cases difficult to fit into any categorical classification. This also affects the role of nuclear medicine (NM) in psychiatry.

\section{Diagnosis in psychiatry}

Today's diagnosis in psychiatry largely relies on the classification of diseases following the criteria of the Diagnostic and Statistical Manual of Mental Diseases (DSM-IV-TR-2000) [1]. This classification enabled better definition of nosological concepts and better communication and understanding between psychiatrists.

On the other hand, DSM-IV does not entirely fit the psychiatric diagnosis due to its rigid application of choice principle (five out of nine symptoms).

There are two major problems in psychiatric diagnosis as a whole:

- diversity of symptoms - depressive episode patients may be either agitated and insomniac, or with psychomotor retardation and hypersomnia.

- overlapping symptoms - (sometimes defined as "co-morbidity"); e.g. 30-60\% of depressive patients may have co-morbid anxiety disorder, whereas $40 \%$ diagnosed with anxiety have [2]

\section{Diagnosis in psychiatry - the role of neuroimaging}

In the same way that the symptoms between different diseases in psychiatry overlap, functional brain research frequently shows

\footnotetext{
Correspondence to: Piotr Lass

Department of Nuclear Medicine, Medical University

ul. Dębinki 7, 80-211 Gdańsk, Poland

Tel/fax: (+48 58) 3492204

e-mail: plass@amg.gda.pl
}

the same pattern of changes across diagnostic borders; on the other hand, many the other tests, e.g. psychological tests, present the same problem as mentioned above; therefore:

The psychiatrist seldom applies to an NM specialist to obtain a diagnosis; instead, a nuclear medicine report will rather confirm, or less frequently exclude, the psychiatrist's diagnosis. Ideally, psychiatric patients should be rescanned after the treatment, and changes in perfusion and/or metabolism discussed between psychiatrist and NM specialist.

\section{Applications of nuclear medicine}

Applications of NM can be divided into:

I. Mostly diagnostic/practical/clinical:

- differential diagnosis of dementia;

- psychiatric sequelae of head trauma, including late whiplash syndrome;

- neuropsychiatric lupus erythematosus.

II. With mixed/indeterminate status:

- psychiatric disorders in parkinsonian syndromes;

- chronic fatigue/myalgic encephalomyelitis.

III. Mostly investigational/experimental:

— other/most receptor studies.

\section{Differential diagnosis of dementia}

There are five potential major roles for neuroimaging with respect to dementia:

- as a cognitive neuroscience research tool;

— for the prediction of which normal or slightly impaired individuals will develop dementia and over what time frame;

- for early diagnosis of Alzheimer's disease (AD) in demented individuals, (sensitivity) and separation of AD from other forms of dementia (specificity);

- for monitoring disease progression;

— for monitoring response to therapy.

\section{Alzheimer's disease - SPECT studies}

Single photon emission-computed tomography (SPECT) of regional cerebral blood flow (rCBF) reaches detecting $A D$ in up to $81 \%$; although the clinical criteria may more sensitive (up to $81 \%$ ), SPECT seems superior in differentiating AD from the other types of dementia (91\% vs. $70 \%$ ) [3]. Applying novel methodological approaches such as means clustering or principal component analysis has improved SPECT accuracies to as much as 98 and $90 \%$, respectively [4] 
In SPECT studies, the typical finding is decreased blood flow in the parietal or temporal lobe; in advanced cases with poor prognosis, decreased blood flow in the frontal lobes is also found. In mild cognitive impairment, rCBF SPECT studies may differentiate patients who will convert to $A D$ from non-converters. Converters show reduced rCBF in bilateral temporoparietal areas and the precunei, compared with non-converters. The logistic regression model reveals that reduced rCBF in the inferior parietal lobule, angular gyrus and precunei has a high predictive value and discriminative ability [5]. The acetazolamide test in rCBF SPECT is helpful to differentiate AD from vascular dementia [6]

Following the therapy with acetyl cholinesterase inhibitors (AChEl), the regional cerebral blood flow increases or remains stable in $A D$ patients with stabilized cognitive performance during therapy, but decreases in non-responders [7]

\section{Alzheimer's disease - metabolic studies}

[(18)F] fluorodeoxyglucose (FDG) possitron emission tomography $(P E T)$ images of $A D$ demonstrate a focally decreased cerebral metabolism especially involving the posterior cingulate and neocortical association cortices, while largely sparing the basal ganglia, thalamus, cerebellum and cortex mediating primary sensory and motor functions.

In a multicentre study comprising 10 PET centres (Network for Efficiency and Standardisation of Dementia Diagnosis, NEST-DD) that employed an automated voxel-based analysis of FDG-PET images, the distinction between controls and $A D$ patients was $93 \%$ sensitive and $93 \%$ specific, and even in very mild dementia (at MMSE 24 or higher) sensitivity was still $84 \%$ at $93 \%$ specificity [8] In very mild $A D$, both FDG-PET and voxel-based morphometry (VBM-MRI) had high accuracies for diagnosis, but FDG-PET showed a slightly higher accuracy than VBM-MRI. A combination of the two techniques will yield a higher diagnostic accuracy in very mild $A D$ by making full use of functional and morphological images [9].

In addition to glucose metabolism, specific tracers for dopamine synthesis ( $\left.{ }^{18} \mathrm{~F}-\mathrm{F}-\mathrm{DOPA}\right)$ and for $\left({ }^{11} \mathrm{C}-\mathrm{MP} 4 \mathrm{~A}\right)$ are of interest for differentiation among dementia subtypes. Cortical acetylcholine esterase activity $(A C h E)$ is significantly lower in patients with $A D$ or dementia with Lewy bodies (DLB) than in age-matched normal controls. In LBD there is also an impairment of dopamine synthesis, similar to Parkinson's disease.

\section{Alzheimer's disease - imaging amyloid deposits}

Pathologically, AD is characterised by the excess accumulation of two types of protein aggregates: amyloid $\beta$-peptide $(A \beta)$ plaques and neurofibrillary tangles.

Amyloid imaging with SPECT is possible with radioiodinated styrylpyridines and hydroxybenzothiazoles [10], but today, practically, PET is the only available $A \beta$ imaging technique with the following agents:

- fluorophore derivative ${ }^{18} \mathrm{~F}-\mathrm{FDDNP}[11]$;

- benzothiazole derivative ${ }^{11} \mathrm{C}-\mathrm{PIB}[12]$

— stilbene derivative ${ }^{11} \mathrm{C}$ SB-13 [13];
— ${ }^{18} \mathrm{~F}$-fluoropegylated diphenylacetylenes [14].

Due to high costs, these modalities remain solely investigational.

\section{Differential diagnosis of dementia - DLBD}

Diffuse Lewy body dementia (DLBD) is the second most common type of degenerative dementia after $A D$ and accounts for 15-20\% of all autopsy-confirmed dementias in old age. Core clinical features are progressive cognitive impairment, visual hallucinations and parkinsonian symptoms and signs. In DLBD there is a pronounced cholinergic deficit, therefore, cholinergic medication gives better results, than in AD. In its early stages, DLBD brings difficulties in differentiation with $A D$.

On rCBF SPECT scanning, patients show hypoperfusion in parietal and occipital, and in frontal lobes, called "horseshoe" signs [15]. Combined studies of MMSE and brain SPECT achieved a high discrimination between DLB and AD with a sensitivity of $81 \%$ and a specificity of $85 \%$, suggesting that this is a useful and practical approach to differentiate DLB from AD [16]. (18)F-FDG PET showed significant glucose metabolic reductions in the temporal, parietal and frontal areas (including the occipital lobe) compared with those in the control group; in contrast, in AD patients, both the hippocampal volume and glucose metabolism were significantly decreased, whereas the occipital volume and metabolism were preserved [17]

\section{Fronto-temporal dementia}

Fronto-temporal dementia (FTD) generally has a presenile onset, behavioural problems dominate the clinical picture and cognitive functions are still relatively intact.

In rCBF SPECT studies, rCBF defect in frontal lobes in the left temporoparietal-occipital discriminates FTD and $A D$ and with a sensitivity of 0.8 and specificity 0.65 . $[18,19]$.

In PET studies in early stages of FTD, the neurodegenerative process was found to be limited to the frontal lobes. During the progression of the disease, the pathological changes pass over the lobar borders and spread into the parietal and temporal cortices [20]. In the late stages a significant hypometabolism is found mostly in extensive prefrontal areas, cingulate gyri, anterior temporal regions and the left inferior parietal lobule. Frontal hypometabolism is usually more prominent in the left hemisphere than in the right — in $79 \%$ of patients [21].

Therefore sociopathy in FTD may result from right frontotemporal dysfunction. In many jurisdictions, FTD patients with sociopathy would not pass legal criteria for "not guilty" due to insanity.

\section{Dementia in Parkinson's disease/ /Parkinsonian syndromes}

Parkinson's disease is a neurodegenerative disorder characterised by progressive damage of the nigrostriatal dopaminergic neurons in the basal ganglia. It accounts for up to $85 \%$ of patients with parkinsonian symptoms.

Parkinsonian syndromes is a broader definition that encompasses other movement disorders with symptoms resembling PD 
and includes progressive supranuclear palsy (PSP), multiple system atrophy (MSA) and corticobasal degeneration.

Confusingly, the symptoms of PD or PS can also be met in patients with essential tremor or secondary to some medications. Therefore, up to $25 \%$ of patients initially diagnosed as PD, later have this diagnosis changed. $20-30 \%$ of patients with PD have dementia; the crucial problem of diagnosing it with, for example MMSE are motor and speech disturbances.

PD patients with dementia show left temporo-parietal hypoperfusion as compared to a group of patients without dementia, which resembles perfusion deficits described in Alzheimer's disease. Hypoperfusion of the left temporal lobe with an increase of rCBF within the left thalamus might be clinically useful in the discrimination of Parkinson's disease patients with dementia from those without cognitive impairment [22].

Another aspect of RN diagnosis aims to differentiate PD and essential tremor by ${ }^{123}$ - $\beta$-CIT or ${ }^{18} \mathrm{~F}$-DOPA uptake and differentiating $P D$ and $P S$-plus syndromes with imaging of $D_{2}$ receptors with 123|-IBZM [23-25].

\section{Post-traumatic disorders}

After head trauma a number of neuropsychiatric symptoms and signs may follow, with considerable difficulties both in the treatment and in medical certification of posttraumatic neuropsychiatric disorders for the purpose of criminal and civil law proceedings.

Functional brain imaging data collected in a resting state can provide objective evidence of brain injury in mild blunt head trauma patients with persistent postconcussive somatic and/or cognitive symptoms, particularly in mild and moderate traumatic brain injury by better sensitivity than MR/CT findings and providing an important exclusion role [26, 27], e.g. young patients following head trauma $97 \%$ with negative rCBF SPECT findings develop no post-traumatic disorder, whereas $95 \%$ with rCBF changes do. RCBF SPECT scanning is particularly useful in imaging blood flow disturbances in basal ganglia following head traumas, as well as in mild brain injury [26-27]. The results of rCBF scanning are important in rehabilitation counselling, medico-legal arguing and the evaluation of the ability of the patient to work. Its limitations overlap diffuse axonal injury (DAl), invisible in PET/SPECT, and in older patients overlapping, for example, atherosclerotic vascular lesions.

\section{Late whiplash syndrome}

The prevalence of whiplash injury is 4 cases per 1000 inhabitants per year, usually following rear-end car collisions. This sometimes results in whiplash-associated disorder (WAD), a controversial condition with largely unknown pathogenetic mechanisms. Only a small proportion (up to $5 \%$ ) of whiplash injury patients develop late whiplash syndrome with cerebral symptoms: headache, dizziness, vertigo and concentration attention and memory disturbances, as well as peripheral symptoms: neck pain, neck rigidity, temporomandibular dysfunction.

Being frequently secondary to traffic, sport and work related accidents, late whiplash syndrome is a major medico-legal prob- lem, of interest not only to physicians, but also to lawyers and insurance companies.

\section{Late whiplash syndrome - triggering Alzheimer's disease?}

In 1997-2003 Otte et al. described an increased parieto/occipital hypoperfusion and hypothesized that LWS may trigger Alzheimer's disease following prolonged hypoperfusion in the cortico-basal region [28-30].

This hypothesis has been heavily criticized by many opponents, eg. Sundström and colleagues, who showed rCBF changes in patients with chronic back pain of non-traumatic origin, but not in those following whiplash injury [31], whereas some data supports it [32]

\section{Neuropsychiatric involvement in systemic lupus erythematosus}

The peak incidence of systemic lupus erythematosus (SLE) occurs between 15 and 40 years of age. Central nervous system (CNS) manifestations have been described in $20-70 \%$ of cases. This dispersion alone illustrates the basic problem of cerebral involvement in SLE, i.e. differentiating organic brain lesion from functional disturbances, influence of medication - particularly steroids, feelings of social rejection following skin changes, etc. Neuropsychiatric manifestations in SLE comprise: migraine, epilepsy and stroke, cognitive dysfunction, mood disorder, anxiety disorder, acute confusion state and psychoses.

Radionuclide studies help to distinguish organic brain lesions and to differentiate from functional/iatrogenic changes. The clinician expects a zero/one answer to the question: is there CNS involvement or not?

As a "golden standard" of neuroimaging in neuropsychiatric systemic lupus erythematosus (NP-SLE) is considered, MRI scanning with white matter hyperintensities $(\mathrm{WMH})$ as a marker of vascular involvement. However, this appears in $30 \%$ of SLE patients without CNS involvement and is absent in the early/intermediate stages of the disease.

As a result, rCBF SPECT scanning is the most sensitive, although not entirely specific for neuroradiological assessment in NP SLE and other loose connective tissue diseases [33]. The higher sensitivity of SPECT, compared with MRI, can be explained by the vasculopathy with microcirculation changes as a major pathological factor in SLE.

${ }^{18}$ FDG PET scanning shows "posterior" type hypometabolism as a typical finding [34].

\section{Schizophrenia}

Schizophrenia is a major source of morbidity worldwide, with a prevalence of about $1 \%$ and significant disablement. RN neuroimaging is focused on receptor research, with few results, mostly indicating disturbances of dopamine in schizophrenia, but this is unlikely to be the whole story. As new ligands are developed, further insights will be gained into the underlying pathology of schizophrenia; new techniques combining functional imaging with genetic studies are likely to not only depict the state of receptor populations, but also concentrate on long-term dynamic changes induced by the illness and its treatment. 


\section{Schizophrenia - the dopamine overactivity hypothesis}

This hypothesis says that increased activity in the dopamine neurotransmitter system is responsible for the positive symptoms of schizophrenia. An increased density of $D_{2}$ receptors was found in post-mortem studies [35]

Early studies revealed a marked increased in $D_{2}$ binding within the striatum. Further studies revealed two distinct families of dopamine receptors: $D_{1}^{-}$like $\left(D_{1}\right.$ and $\left.D_{5}\right)$ and $D_{2}{ }^{-}$like $\left(D_{2}, D_{2}, D_{4}\right)$. It could be that the antipsychotic drug selectivity could be due to preferential binding of dopamine $\mathrm{D}_{2}$ like receptors.

For measuring dopamine synthesis and transport in presynaptic function for the former, the most commonly used tracers are 6-[(18)F]FDOPA and 6-[(18)F]FMT, whereas for the latter, several (11)C/(18)F-labelled tropane analogues are being clinically used. Postsynaptically, dopamine exerts actions through several subtypes of the dopamine receptor [36].

In schizophrenia, dopamine studies of receptor competition of ${ }^{123}$ I-IBZM, a dopamine $D_{2}$ ligand with dopamine, showed that not only resting dopamine levels matter, but also suggested that schizophrenia is a disorder of dopamine dysregulation in different parts of the brain. In treatment follow-up, increasing $D_{2}$ receptor occupancy on the $2^{\text {nd }}$ SPECT was a predictive factor for the relapse; therefore, $D_{2}$ receptor occupancy and its changes during quetiapine therapy is thought to be related to the prognosis of the treatment efficacy [37]

\section{Schizophrenia - $5 H T$ receptors}

An interest in the role of $5 \mathrm{HT}_{2 \mathrm{a}}$ receptors in schizophrenia was aroused by the observation that serotoninergic agents, such as LSD, led to hallucinations. Initial SPECT and PET studies showed a very high occupancy of $5 \mathrm{HT}_{2 \mathrm{a}}$ receptors for many atypical antipsychotic drugs including clozapine, olanzapine and risperidone but not typical ones like haloperidol and chlorpromazine. The involvement of $5 \mathrm{HT}_{2 \mathrm{a}}$ receptors was undermined by the fact that its pure antagonists did not have any antipsychotic effect. More recent papers on $5 \mathrm{HT}_{1 \mathrm{a}}$ have shown increased binding in schizophrenics in the left mediotemporal cortex, but the meaning of this is unclear [38]. The current view is that changes in this receptor population are unlikely to be a causal factor in schizophrenia.

\section{Schizophrenia - glutamate and NMDA receptors}

N-Methyl-D-aspartate (NMDA) receptors are calcium-permeable glutamate receptors that play putative roles in learning, memory and excitotoxicity. There has been much interest over the years in the NMDA receptors in schizophrenia, as certain blockers of NMDAR such as PCP and ketamine lead to transient drug-induced symptoms very similar to those reported by schizophrenic patients. The first in vivo evidence of an NMDA receptor deficit in medication-free schizophrenic patients was published in 2006 [39]

\section{Mood disorders}

Mood disorders are amongst the most prevalent in modern society and have an important socio-economic impact.

Diagnosing depression is based upon fulfilling five criteria of the American Psychiatric Association/DSM-IV, including at least "depressed mood" or "diminished interest of pleasure" during the same 2-week period. The presence of manic or hypomanic episodes further specifies whether the disorder is unipolar or bipolar.

The course specifiers describe the severity of the last episode, from mild to severe, and the course of disorders, e.g. recurrent, with seasonal pattern or with rapid cycling pattern; other specifiers describe atypicality, postpartum onset and the presence of catatonic features or chronicity.

\section{Brain perfusion and metabolism studies in depressive disorders}

Early studies by Baxter et al. described a globally decreased supratentorial brain activity in bipolar disorders. With metabolic rate increasing while going from depression to euthymic or manic state, further studies showed a significant left-right prefrontal asymmetry, resolving after treatment. Hypoperfusion in recurrent depressive disorders was considerably greater (nearly significant) in comparison with the first depressive episodes [40]

Further studies refined the findings of prefrontal hypoperfusion/metabolic hypoactivity, as the most important delineating those findings to areas of the prefrontal cortex and its functionally separate areas: dorsolateral (DLPFC), the orbito-frontal and anterior cingulate, left amygdala, parahippocampal gyrus [41, 42], which with subcortical circuits have separate behavioural functions: DLPFC mediating executive functions, orbito-frontal object-affect associations and anterior cingulate mediating motivation.

\section{Mood disorders - treatment effects}

Nearly all available antidepressants have been studied. Normalisation of the frontal hypoperfusion/ hypometabolism and/or asymmetry appears to be the most replicative finding [43, 44].

In many studies a pattern of cortical flow/metabolism increases and limbic/paralimbic decreases was seen in association with chronic treatment, which suggest primary subcortical and limbic effects of pharmacological treatment, with neocortical effects as secondary [45]

Electroconvulsive therapy (ECT), a powerful tool where pharmacological intervention fails, initially reduces the CBF and metabolism in the short term, suggesting the reduction of neural activity; in the long-term, normalising perfusion/metabolism in depressed patients $[43,46]$. However, ECT flow/metabolism results may be atypical, as this therapy is mostly applied in treatmentresistant patients.

Repetitive transcranial magnetic stimulation (rTMS) of the prefrontal cortex may be useful in refractory cases. High-frequency rTMS of the left prefrontal cortex and possibly of the opposite physiological effects low frequency rTMS of the right prefrontal cortex produce a significant decrease in Hamilton depressing scale scores; it increases the metabolic rate in the prefrontal cortex, amygdala, basal ganglia, hippocampus and cerebellum; posttreatment a decrease in cingulate [47].

Vagal nerve intermittent electrical stimulation, used mostly in epilepsy treatment, also reduces depressive symptoms. rCBF changes share features with changes of rCBF previously associated with the administration of selective serotonin reuptake inhibitors. Similarities to other brain-stimulation strategies in antidepressant treatment were less pronounced [48]. 


\section{Radioligand receptor studies in depression}

These studies are limited mainly by the availability of suitable ligands. Currently available ligands allow the investigation of 5- $\mathrm{HT}_{1 \mathrm{~A}}$ [11C-WAY-100635], 5- $\mathrm{HT}_{2 \mathrm{~A}}\left[{ }^{123} \mathrm{I}\right.$-ketanserin, ${ }^{18} \mathrm{~F}$-altanserin, and ${ }^{11} \mathrm{C}$-methylpiperone] and $\mathrm{D}_{2}$ receptors [ ${ }^{123|-| B Z M}$. The results are rather discordant $[49,50]$.

Most, albeit not all, studies find an increased $5-\mathrm{HT}_{2 \mathrm{~A}}$ binding after treatment, the same effect in $D_{2}$ binding and a decrease in $\mathrm{HT}_{1 \mathrm{~A}}$ binding.

The current status of ligand studies in depression does not yet allow for the tailoring of the pharmacotherapeutic status in individual patients. They allow at least some agreement as to the effect of antidepressant agents on the HT and dopamine receptors and on serotonin transporters.

There are two conclusions about radionuclide studies in depression: good news: brain imaging is a powerful tool to explore various aspects of brain function in depression; bad news: the clinical psychiatrist should not ask the nuclear medicine psychiatrist to confirm the diagnosis of depression in his patients - hypofrontality is quite an unspecific finding. Performing functional imaging, however, should stimulate both specialists to look at functional abnormalities on the scan and to link them to the patients behavioural abnormalities and/or symptoms.

Future research should be devoted to finding the basics of treatment regimens and finding predictors of treatment response.

\section{Obsessive-compulsive disorder}

Obsessive-compulsive disorder (OCD) is characterised by repetitive thoughts, impulses, images or behaviours. The obsessions or compulsions interfere significantly with the person's normal life as well as occupational and social functioning.

Neuroimaging studies have proved that the evidence of the dysfunction of the fronto-subcortical circuit might be involved in the pathophysiology of OCD. In SPECT and PET studies hyperactivities of those circuits, including orbito-frontal cortex, anterior cingulate and/or basal ganglia, have been a consistent finding [51]

Serotonin transporter studies indicate a reduced serotonergic input into the fronto-subcortical circuits in $O C D$, thereby diminishing the inhibitory regulation of serotonin in these circuits [52].

Fluvoxamine treatment significantly improves clinical symptoms and increases $D_{2}$ ligand [(11)C]-raclopride binding potential (BP) in the basal ganglia of OCD patients. (Chronic treatment with fluvoxamine induces a slight but significant increase in striatal [(11)C]-raclopride binding of previously drug-naive OCD patients [53].

Although present imaging techniques have limitations as a means of brain dysfunction in $\mathrm{OCD}$, nuclear neuroimaging may be used as an objective tool and as a way to predict a response to the treatment.

\section{Eating disorders}

Eating is part of basic human behaviour. Therefore, eating disorders are relative rare within the domain of psychiatry. There are two major pathological entities: anorexia nervosa and bulimia nervosa. On the other hand, a separate major issue is the investigation of the CNS in binge-eating and obesity: a major epidemic problem. Many obese patients probably suffer from binge-eating disorders, but this is certainly not always objectified and is even overlooked.

Functional brain imaging in eating disorders has been available for more than a decade. Generally there is an enormous differentiation between studies, and they may be secondary to methodological variations. Therefore they are of limited, or even no clinical value, at least at present, although they may give some guidance to future pharmacological studies.

\section{Anorexia nervosa}

Anorexia nervosa (AN) is a devastating and life-threatening disease. Among females, its prevalence is approximately 0.27 $1 \%$ [54]. There are two subtypes: in the RAN subtype, dieting, fasting or excessive exercise is used to achieved weight loss; the binge-eating/purging type involves a combination of binge eating and purging by means of self-induced vomiting and/or misuse of laxatives, diuretics and enemas.

Patients with anorexia nervosa show either hyperperfusion in the frontal, fronto-temporal and parietal cortex or hypoperfusion in the anterior cingulate. Activation tests show an rCBF increase in the left inferior frontal lobes. Following treatment, changes of rCBF in the right DLPFC, ACC, MPFC, PCC and precuneus are related to the $A N$ recovery process and might be associated with the improvement of interoceptive awareness [55].

Receptor [ ${ }^{18} \mathrm{~F}$-altanserin] $\mathrm{HT}_{2 \mathrm{~A}}$ studies showed reduced tracer binding with altered neurotransmission during recovery. The question remains, whether it is a cause or consequence of $\mathrm{AN}$.

There are two basic technical problems in the assessment of AN patients: rCBF changes are either small and space-restricted or diffuse and unspecific. This requires either good quality statistical parametric mapping (SPM) or activation paradigms utilising food stimuli in a well-standardized environment.

\section{Bulimia nervosa}

Bulimia (Greek — bull hunger) is generally considered a condition in which the subject engages in recurrent binge eating followed by an intentional purging. This purging is done in order to compensate for the excessive intake of food and to prevent weight gain. Purging typically takes the form of vomiting; inappropriate use of laxatives, enemas and other medication and/or excessive physical exercise. Historically, it was initially considered a variant of anorexia nervosa.

SPECT studies showed decreased right inferior frontal and left temporal lobes. This may suggest hypoactivity of the putative feeding suppression mechanism in the frontal lobe, resulting in hyperphagia. Alterations in $\mathrm{rCBF}$ during the ill state of $\mathrm{BN}$ may be a state-related phenomenon that remits with recovery [56].

PET study data showed - contrary to depressive patients - maintained basal ganglia metabolism. Some PET studies showed lower rCMRGlu patterns similar to those of obsessive-compulsive disorder symptoms.

Receptor studies suggest decreased serotonergic transmission in bulimia nervosa. Studies using PET with serotonin specific radioligands implicate alterations of $5-\mathrm{HT} 1 \mathrm{~A}$ and $5-\mathrm{HT} 2 \mathrm{~A}$ receptors and the $5-\mathrm{HT}$ transporter. Alterations of these circuits may affect mood and impulse control as well as the motivating and hedonic aspects of feeding behaviour [57]. 


\section{Obesity}

Obesity is an epidemic of our age, at least in some countries; therefore, functional brain studies in obesity seem very promising. $\mathrm{rCBF}$ SPECT studies in obese women showed higher CBF in the right parietal and temporal cortices during food exposure than in control conditions. In addition, in obese women the activation of the right parietal cortex was associated with an enhanced feeling of hunger when looking at food. No such changes or associations were seen in normal-weight women [58].

In PET studies, higher metabolic activity was shown in the area of the parietal cortex where the somatosensory maps of the mouth, lips and tongue are located, suggesting the role of a reward component in the aetiology of obesity. Another study showed the involvement of enhanced sensitivity of the frontal regions following food stimuli, suggesting their specific response.

Receptor studies with ${ }^{11} \mathrm{C}$-raclopride showed the negative correlation of $\mathrm{BMI}$ and $\mathrm{D}_{2}$ receptor availability [59].

\section{Social anxiety disorder}

Social anxiety disorder (SAD) is characterised by the fear of social interaction and performance situations. A person fears that he or she will act in a way that will be humiliating or embarrassing [DSM-IV]. In the course of the disease, secondary depression or subsequent alcohol abuse and dependence may develop.

rCBF SPECT scanning data are similar to those of other anxiety disorders and are consistent with previous work demonstrating the importance of limbic circuits in this spectrum of disorders. These play a crucial role in cognitive-affective processing, are innervated by serotonergic neurons, and changes in their activity during serotonergic pharmacotherapy seem crucial [60].

Serotonin receptor studies showed that the lower $5-\mathrm{HT}_{1 \mathrm{~A}}$ binding in the amygdala and mesiofrontal areas of SAD patients was consistent with a previous PET study in healthy volunteers showing an inverse correlation between $5-\mathrm{HT}_{1 \mathrm{~A}} \mathrm{BP}$ and state anxiety and other human PET studies in patients with panic disorders showing reduced $5-\mathrm{HT}_{1 \mathrm{~A}}$ binding, thus corroborating the potential validity of $5-\mathrm{HT}_{1 \mathrm{~A}}$ receptors as targets in the treatment of human anxiety disorders [61].

Dopamine receptor studies showed that striatal dopamine reuptake site densities were markedly lower in patients with social phobias than in the age- and gender-matched comparison subjects These results indicate that social phobia may be associated with a dysfunction of the striatal dopaminergic system [62].

\section{Functional brain imaging in court}

PET and SPECT studies are increasingly used as tools in forensic medicine, particularly in the USA since the start of the nineties. The mental disorders which attract the most legal attention are those where a connection is made to an injurious stimuli, e.g. post-traumatic brain injury or post-traumatic stress disorder. The brain changes are not clear in all patients; therefore, functional neuroimaging cannot be safely used to make diagnosis of psychiatric disorders or to exclude/confirm psychiatric disorders, so its use is limited to that of an auxiliary tool together with $\mathrm{CT}, \mathrm{MRI}$ and psychometric testing, although some specific reports exist $[63,64]$
In order to be accepted as evidence, the Supreme Court of the USA ruled that the following elements should be taken into account assessing the diagnostic method [65]:

- can the particular method be tested?

- are the known/potential pitfalls of the method established?

- is there sufficient scientific evidence about the method?

- is there wide acceptance of the method in the scientific world?

In most cases, PET and SPECT fulfil the above-mentioned demands, at least partly, due to the scarce number of controlled studies, low sensitivity and specificity, and insufficient standardisation. The have, therefore, used in only a few situations: traumatic brain injury following car accidents or criminal attacks, in confirming/excluding brain injury in victims with unspecific complaints such as persistent headaches, amnesia emotional disorders etc., organic basic brain dysfunction e.g. frontal syndrome in suspects, or post-traumatic stress disorder (PTSD) in war veterans.

\section{Post-traumatic stress disorder}

Post-traumatic stress disorder (PTSD) is a complex psychobiological disorder which develops in the aftermath of severe and/ /or life-threatening trauma (coma, burns, sexual abuse). Functional imaging in PTSD is in its infancy, but the body of evidence is growing, and through these insights PTSD has changed from "traumatic neurosis" to a biologically based psychological disorder. This anxiety disorder is most likely associated with changes in neural circuitry involving the frontal and limbic systems. The altered metabolism in these brain structures after a traumatic event is correlated to PTSD. Developments in the field of neuroimaging have allowed researchers to look at the structural and functional properties of the brain in PTSD [66].

Neuroimaging studies are usually performed as emotional activation studies, utilising e.g. combat-related sounds, images of a great fire, etc. [67, 68]; therefore, such studies are sometimes difficult to perform for ethical reasons.

Emotional activation studies demonstrate flow/metabolic activation most frequently in the precentral gyrus, posterior cingulate, amygdala and cerebellum, while deactivation is seen in the middle temporal gyrus, and inferior and middle frontal and parietal region.

Preliminary data show the circuits in the brain connecting regions of the medial prefrontal, medial temporal, frontal and parietal cortices. The future of such studies will probably help in understanding what makes some people more vulnerable to PTSD and how treatment influences and predicts the changes we see in brain imaging studies.

\section{Brain function during hypnosis}

Hypnosis may be defined as a state of focused attention, concentration and inner absorption with a relative suspension of peripheral awareness.

PET studies demonstrated a vast activation in the occipital parietal, precentral prefrontal and cingulate cortex. This suggests that the hypnotic state does not rely on simple evocation of episodic memory but a reminiscence of mental imagery [69].

PET studies have given neuroimaging support for the nociceptive effect of hypnosis; the hypnotic state significantly enhances 
the functional modulation between the midcingulate cortex and the large neural network involved in sensory, affective, cognitive and behavioural aspects of nociception [70]. In fibromyalgic patients under hypnosis, the cerebral blood-flow was bilaterally increased in the orbitofrontal and subcallosal cingulate cortices, the right thalamus and the left inferior parietal cortex, and was decreased bilaterally in the cingulate cortex. The observed bloodflow pattern supports notions of a multifactorial nature of hypnotic analgesia, with interplay between cortical and subcortical brain dynamics [71].

\section{Impulsive aggression and suicidal behaviour}

Impulsive aggressive behaviour including deliberate self-harm and impulsive aggression towards others as well as suicidal behaviour is a major problem in healthcare. It is believed that its anatomical correlates are the prefrontal and temporal limbic regions and serotoninergic dysfunction plays a crucial role.

In impulsitivity, PET studies indicate that the impulsivity and chronic stress are associated with amphetamine-induced stria-tal dopamine release. In PET studies with high specific activity, [11C] raclopride was associated with blunted right ventral striatal DA release. Dopamine release was greater in low vs. high impulsivity subjects under conditions of low or moderate stress [72].

In suicidal behaviour, direct in vivo functional imaging with PET or SPECT demonstrated a reduction in the 5-HT2A binding index in suicide attempts by anxious and depressed suicide attempters and an increase in 5-HT2A binding in impulsive suicide attempters [73]. The importance of such studies is two-fold: first this may increase insight into the pathophysiology of impulsive aggression; secondly this may enable the development of new pharmacological approaches.

\section{Hysteria and catatonia}

\section{Hysteria (conversion disorder)}

The neural concomitants of hysteria and catatonia remain largely unknown. Hysteria, or conversion disorder, is defined as a psychiatric illness the symptoms or deficits of which, affecting voluntary motor or sensory function, cannot be explained by a neurological or general medical condition.

Functional neuroimaging has revealed selective decreases in the activity of frontal and subcortical circuits involved in motor control during hysterical paralysis, decreases in somatosensory cortices during hysterical anaesthesia, or decreases in the visual cortex during hysterical blindness [74]. The inhibition of normal neural networks seem to also be a common marker to tunnel vision or hysterical deafness.

\section{Catatonia}

In catatonic patients, SPECT and PET studies showed dysfunctions of prefrontal and parietal cortices that possibly account for its motor, affective and behavioural disorders. Dysfunctions in the prefrontal cortex could account for some affective disturbances found in catatonia, whereas dysfunction in parietal lobes might participate in the disturbances of executive task planning.
In akinetic catatonia, SPECT and PET studies showed dysfunctions of the medial, prefrontal cortex, lateral parietal cortex and precuneus, which are known to be involved in conscious awareness [75].

The studies mentioned support the argument for the existence of a neural network of conscious awareness that may be disturbed in patients with stuporous catatonia.

\section{Sleep disorders}

One-third of our lives are occupied by sleep. The utilization of nuclear medicine in sleep disorders is still in its infancy; this may be, however, a promising area for future development.

In healthy sleep there is an increase in function in the limbic and anterior paralimbic cortex in REM sleep and a decrease in the function of the higher cortical regions in known thalamocortical networks during NMREM sleep. Serotonin $5 \mathrm{HT} 1 \mathrm{~A}$ receptor PET studies showed a significant increase in their ligand-[18F]MPPF binding in sleep compared to wakefulness in the whole brain and all regions of interest examined: temporal cortex, mesial temporal region and cingulate cortex [76]

There is a collection of papers on diverse sleep disorders such as: sleep deprivation, insomnia, dyssomnia, narcolepsy, sleep apnoea and sleepwalking.

Perfusion abnormalities in patients with REM behaviour disorder are located in the brainstem, striatum and cortex. These abnormalities are consistent with the anatomic metabolic profile of Parkinson's disease [77]

In primary insomnia a pattern of hypoperfusion in the frontal medial, occipital and parietal cortices was found with particular deactivation in the basal ganglia [78]

In narcolepsy SPM analysis of the brain, SPECT showed hypoperfusion of the bilateral anterior hypothalami, caudate nuclei, and pulvinar nuclei of the thalami, parts of the dorsolateral/ventromedial prefrontal cortices, parahippocampal gyri and cingulate gyri in narcoleptics, as well as reduced cerebral perfusion in subcortical structures and cortical areas in narcoleptics. The distribution of abnormal cerebral perfusion is concordant with the pathway of the cerebral hypocretin system and may explain the characteristic features of narcolepsy, i.e. cataplexy, emotional lability and attention deficit [79]

In sleepwalking, activation of thalamocingulate pathways and persisting deactivation of other thalamocortical arousal systems was found [80].

\section{Conclusions}

As shown above, there are few practical applications of nuclear medicine due to low specificity and low spatial resolution, although in the aspect of functional imaging it is still superior to $\mathrm{CT} / \mathrm{MRI}$, even in their functional modalities.

On the other hand, its investigational potential is still growing, as there is no imaging technique in sight which could replace metabolic and receptor studies, and also because the scope of functional imaging in psychiatric diseases is spreading from its traditional applications, like dementia or depression, towards many poorly investigated fields e.g. hypnosis, suicidal behaviour or sleep disorders. 


\section{References}

1. DSM-IV-TR (Diagnostic and statistical manual of mental disorders DSM-IV-TR). American Psychiatric Association 2000, Washington DC USA.

2. Audenaert K, Otte A, Peremans K, van Heeringen C, Dierckx R. Functional imaging and functional psychopathology: an introductory chapter. Nuclear medicine in psychiatry. Springer, Berlin-Heidelberg 2004 $1-9$.

3. Dougall NJ, Bruggink S, Ebmeier KP. Systematic review of the diagnostic accuracy of 99mTc-HMPAO-SPECT in dementia. Am J Geriatr Psychiatry 2004; 12: 554-570.

4. Pagani M, Salmaso D, Borbely K. Optimisation of statistical methodologies for a better diagnosis of neurological and psychiatric disorders by means of SPECT. Nucl Med Rev Cent East Eur 2005; 8:140-149 .

5. Hirao K, Ohnishi T, Hirata Y et al. The prediction of rapid conversion to Alzheimer's disease in mild cognitive impairment using regional cerebral blood flow SPECT. Neuroimage 2005; 28: 1014-1021.

6. Pávics L, Grûnwald F, Reichmann K et al. rCBF SPECT and the acetazolamide in the evaluation of dementia. Nucl Med Rev Cent East Eur 1998; 1: 13-19.

7. Shimizu S, Hanyu H, Iwamoto T, Koizumi K, Abe K. SPECT follow-up study of cerebral blood flow changes during Donepezil therapy in patients with Alzheimer's disease. J Neuroimaging 2006; 16: 16-23.

8. Herholz K. PET studies in dementia. Ann Nucl Med 2003; 17: 79-89

9. Kawachi T, Ishii K, Sakamoto $S$ et al. Comparison of the diagnostic performance of FDG-PET and VBM-MRI in very mild Alzheimer's disease. Eur J Nucl Med Mol Imaging 2006; 33: 801-809.

10. Qu W, Kung MP, Hou C, Benedum TE, Kung HF. Novel styrylpyridines as probes for SPECT imaging of amyloid plaques. J Med Chem 2007 50: 2157-2165.

11. Nordberg A. Amyloid imaging in Alzheimer's disease. Curr Opin Neurol 2007; 20: 398-402

12. $\mathrm{Ng} \mathrm{S}$, Villemagne VL, Berlangieri $\mathrm{S}$ et al. Visual assessment versus quantitative assessment of 11C-PIB PET and 18F-FDG PET for detection of Alzheimer's disease. J Nucl Med 2007; 48: 547-552.

13. Kung MP, Hou C, Zhuang ZP, Skovronsky D, Kung HF. Binding of two potential imaging agents targeting amyloid plaques in postmortem brain tissues of patients with Alzheimer's disease. Brain Res 2004 1025: 98-105

14. Chandra R, Oya S, Kung MP, Hou C, Jin LW, Kung HF. New diphenylacetylenes as probes for positron emission tomographic imaging of amyloid plaques. J Med Chem 2007 17: 2415-2423.

15. Brockhuis B, Sławek J, Wieczorek D et al. Cerebral blood flow changes in patients with dementia with Lewy bodies (DLB). A study of 6 cases. Nucl Med Rev Cent East Eur. 2006; 9: 114-118.

16. Hanyu $\mathrm{H}$, Shimizu S, Hirao $\mathrm{K}$ et al. Differentiation of dementia with Lewy bodies from Alzheimer's disease using Mini-Mental State Examination and brain perfusion SPECT. J Neurol Sci 2006; 250: 97-102.

17. Ishii K, Soma T, Kono AK et al. Comparison of regional brain volume and glucose metabolism between patients with mild dementia with Lewy bodies and those with mild Alzheimer's disease. J. Nucl Med 2007; 48: 704-711.

18. Charpentier $P$, Lavenu I, Defebvre $L$ et al. Alzheimer's disease and frontotemporal dementia are differentiated by discriminant analysis applied to (99m)Tc-HMPAO SPECT data. J Neurol Neurosurg Psychiatry 2000; 69: 661-663.

19. McNeill R, Sare GM, Manoharan M et al. Accuracy of single-photon emission computed tomography in differentiating frontotemporal dementia from Alzheimer's disease. J Neurol Neurosurg Psychiatry 2007 78: 350-355

20. Diehl-Schmid J, Grimmer T, Drzezga A et al. Decline of cerebral glu- cose metabolism in frontotemporal dementia: a longitudinal 18F-FDG-PET-study. Neurobiol Aging 2007; 28: 42-50.

21. Jeong $Y$, Song YM, Chung PW et al. Correlation of ventricular asymmetry with metabolic asymmetry in frontotemporal dementia. J Neuroradiol 2005; 32: 247-254

22. Derejko M, Slawek J, Wieczorek D, Brockhuis B, Dubaniewicz M, Lass P. Regional cerebral blood flow in Parkinson's disease as an indicator of cognitive impairment. Nucl Med Commun 2006; 27: 945-951.

23. Slawek J. Cerebral blood flow SPECT scanning in cortico-basal degeneration. Nucl Med Rev Cent East Eur 1999; 2: 85-86.

24. Gerasimou GP, Aggelopoulou TC, Costa DC, Gotzamani-Psarrakou A Molecular imaging (SPECT and PET) in the evaluation of patients with movement disorders. Nucl Med Rev Cent East Eur 2006; 9: 147-153 .

25. Van Laere K, Casteels $C$, De Ceuninck L et al. Dual-tracer dopamine transporter and perfusion SPECT in differential diagnosis of parkinsonism using template-based discriminant analysis. J Nucl Med 2006; 47: 384-392.

26. Lewine JD, Davis JT, Bigler ED et al. Objective documentation of trau matic brain injury subsequent to mild head trauma: multimodal brain imaging with MEG, SPECT, and MRI. J Head Trauma Rehabil. 2007; 22: 141-55.

27. Abu-Judeh HH, Parker R, Aleksic S et al. SPECT brain perfusion findings in mild or moderate traumatic brain injury. Nucl Med Rev Cent East Eur 2000; 3: 5-11.

28. Otte A, Ettlin TM, Fierz L, Kischka U, Mürner J, Müller-Brand J. Brain perfusion in 136 patients with chronic symptoms after distortion of the cervical spine using single photon emission computed tomography, technetium-99m-HMPAO and technetium-99m-ECD: a controlled study. J Vasc Invest 1997; 3: 1-7.

29. Otte A, Nitsche E, Mueller Brand J. Letter to Editor. Reply. J Nucl Med 1998; 39: 329-930.

30. Otte A. Functional neuroimaging in late whiplash syndrome: a denied diagnostic procedure of political concern? Hellenic Journal of Nuclear Medicine 2003; 6: 86-87.

31. Sundström T, Guez M, Hildingsson C, Toolanen G, Nyberg L, Riklund K Altered cerebral blood flow in chronic neck pain patients but not in whiplash patients: a 99mTc-HMPAO rCBF study. Eur Spine J 2006; 15: 1189-1195.

32. Lorbeyboym M, Gilad, R, Gorin V, Sadeh M, Lampl Y. Late whiplash syndrome: correlation of brain SPECT with neuropsychological tests and P300 event-related potential. J Trauma 2002; 52: 521-526.

33. Lass $P$, Krajka-Lauer J, Homziuk $M$ et al. Cerebral blood flow in Sjogren's syndrome using 99Tcm-HMPAO brain SPET. Nucl Med Commun 2000; 21: 31-35

34. Weiner SM, Otte A, Schumacher M et al. Diagnosis and monitoring of central nervous system involvement in systemic lupus erythematosus: value of F-18 fluorodeoxyglucose PET. Ann Rheum Dis 2000; 59 377-385

35. Seeman P, Ulpian C, Bergeron C et al. Bimodal distribution of dopamine receptor density in grains of schizophrenics. Science 1984; 225 728-731.

36. Elsinga $\mathrm{PH}$, Hatano $\mathrm{K}$, Ishiwata $\mathrm{K}$. PET tracers for imaging of the dopaminergic system. Curr Med Chem 2006; 13: 2139-2153.

37. Pávics L, Szekeres G, Ambrus E et al. The prognostic value of dopamine receptor occupancy by [123I]IBZM-SPECT in schizophrenic patients treated with quetiapine. Nucl Med Rev Cent East Eur 2004; 7 129-133.

38. Tauscher J, Kapur S, Verhoeff NP et al. Brain serotonin 5-HT(1a) receptor binding in schizophrenia measured by positron emission tomography and [11C]-WAY-100635. Arch Gen Psychiatry 2002; 59 514-520.

39. Pilowsky LS, Bressan RA, Stone JM, Erlandsson K, Mulligan RS 
Krystal JH, Ell PJ. First in vivo evidence of an NMDA receptor deficit in medication-free schizophrenic patients. Mol Psychiatry 2006; 11: 118-119.

40. Banaś $A$, Lass $P$, Straniewska D. Single photon emission computed tomography (SPECT) for the diagnosis of depressive disorders, neurotic and eating disorders. Psychiatr Pol 2005; 39: 497-507.

41. Videlbech P. PET measurements of brain glucose metabolism and blood flow in major depressive disorder: a clinical review. Acta Psychiatrica Scand 2000; 101: 11-20.

42. Perico CA, Skaf CR, Yamada A et al. Relationship between regional cerebral blood flow and separate symptom clusters of major depression: a single photon emission computed tomography study using statistical parametric mapping. Neurosci Lett 2005; 26; 265-270.

43. Kohn $\mathrm{Y}$, Freedman N, Lester $\mathrm{H}$ et al. 99mTc-HMPAO SPECT Study of Cerebral Perfusion After Treatment with Medication and Electroconvulsive Therapy in Major Depression. J Nucl Med 2007; 48: 1273-1278.

44. Kocmur M, Milcinski M, Budihna NV. Evaluation of brain perfusion with technetium-99m bicisate single-photon emission tomography in patients with depressive disorder before and after drug treatment. Eur J Nucl Med 1998; 25: 1412-1414

45. Davies J, Lloyd KR, Jones IK, Barnes A, Pilowsky LS. Changes in regional cerebral blood flow with venlafaxine in the treatment of major depression. Am J Psychiatry 2000; 160: 374-376.

46. Awata S, Konno M, Kawashima R et al. Regional cerebral blood flow abnormalities in late-life depression following response to electroconvulsive therapy. Psychiatry Clin Neurosci 2002; 1: 31-40.

47. Speer AM, Kimbrell TA, Wassermann EM et al. Opposite effects of high- and low-frequency rTMS on regional blood activity in depressed patients. Biol Psychiatry 2000; 48: 1133-1141.

48. Zobel A, Joe A, Freymann $\mathrm{N}$ et al. Changes in regional cerebral blood flow by therapeutic vagus nerve stimulation in depression: an exploratory approach. Psychiatry Res 2005; 139: 165-179.

49. Kugaya A, Sanacora G, Staley JK et al. Brain serotonin transporter availability predicts treatment response to selective serotonin reuptake inhibitors. Biol Psychiatry 2004; 56: 497-502.

50. Cavanagh J, Patterson J, Pimlott S et al. Serotonin transporter residual availability during long-term antidepressant therapy does not differentiate responder and nonresponder unipolar patients. Biol Psychiatry 2006; 59: 301-308

51. Lacerda AL, Dalgalarrondo P, Caetano D, Camargo EE, Etchebehere EC, Soares JC. Elevated thalamic and prefrontal regional cerebra blood flow in obsessive-compulsive disorder: a SPECT study. Psychiatry Res 2003; 30: 125-134.

52. Hasselbalch SG, Hansen ES, Jakobsen TB, Pinborg LH, Lønborg JH Bolwig TG. Reduced midbrain-pons serotonin transporter binding in patients with obsessive-compulsive disorder. Acta Psychiatr Scand 2007; 115: 388-394.

53. Moresco RM, Pietra L, Henin M et al. Fluvoxamine treatment and D2 receptors: a pet study on OCD drug-naive patients. Neuropsychopharmacology 2007; 32: 197-205.

54. Keski-Rahkonen A, Hoek HW, Susser ES et al. Epidemiology and course of anorexia nervosa in the community. Am J Psychiatry. 2007 164: 1259-1265

55. Matsumoto R, Kitabayashi Y, Narumoto J et al. Regional cerebral blood flow changes associated with interoceptive awareness in the recovery process of anorexia nervosa. Prog Neuropsychopharmacol Biol Psychiatry 2006; 30: 1265-1270.

56. Frank GK, Kaye WH, Greer P, Meltzer CC, Price JC. Regional cerebral blood flow after recovery from bulimia nervosa. Psychiatry Res 2000 100: 31-39.

57. Kaye WH, Frank GK, Bailer UF et al. Serotonin alterations in anorexia and bulimia nervosa: new insights from imaging studies. Physiol Behav 2005; 85: 73-81.

58. Karhunen LJ, Lappalainen RI, Vanninen EJ, Kuikka JT, Uusitupa MI.
Regional cerebral blood flow during food exposure in obese and normal-weight women. Brain 1997; 120: 1675-1684.

59. Wang GJ, Volkow ND, Logan J et al. Brain dopamine and obesity Lancet 2001; 357: 354-357.

60. Carey PD, Warwick J, Niehaus DJ et al. Single photon emission computed tomography (SPECT) of anxiety disorders before and after treatment with citalopram. BMC Psychiatry 2004; 4: 30

61. Lanzenberger RR, Mitterhauser M, Spindelegger C et al. Reduced serotonin-1A receptor binding in social anxiety disorder. Biol Psychiatry 2007; 61: 1081-1089.

62. Tiihonen J, Kuikka J, Bergström K, Lepola U, Koponen H, Leinonen E. Dopamine reuptake site densities in patients with social phobia. Am J Psychiatry 2001; 2: 239-242.

63. Piskunowicz M, Lass, P, Krzyżanowski M, Studniarek M. The usefulness of CBF brain SPECT in forensic medicine. A description of four cases. Nucl Med Rev Cent East Eur. 2001; 4: 47-50.

64. Piskunowicz M, Krzyżanowski M, Lass P, Bandurski T. The usefulness of CBF brain SPECT in forensic medicine: the civil law code cases. A description of four cases. Nucl Med Rev Cent East Eur 2003; 6: 45-47.

65. Daubert versus Merrell Dow Pharmaceuticals. U.S. Supreme Court. 113 Supreme Court 1993:2786; US courts sentences available at web page: http://www.findlaw.com/casecode/supreme.html.

66. Francati V, Vermetten E, Bremner JD. Functional neuroimaging studies in posttraumatic stress disorder: review of current methods and findings. Depress Anxiety 2007; 24: 202-218.

67. Lindauer RJ, Booij J, Habraken JB et al. Cerebral blood flow changes during script-driven imagery in police officers with posttraumatic stress disorder. Biol Psychiatry 2004; 56: 853-861.

68. Peres JF, Newberg AB, Mercante JP et al. Cerebral blood flow changes during retrieval of traumatic memories before and after psychotherapy: a SPECT study. Psychol Med 2007; 9: 1-11.

69. Kosslyn SM, Ganis G, Thompson WL. Neural foundations of imagery Nat Rev Neurosci 2001; 2001: 635-642.

70. Faymonville ME, Boly M, Laureys S. Functional neuroanatomy of the hypnotic state. J Physiol Paris 2006; 99: 463-469.

71. Wik G, Fischer H, Bragee B, Finer B, Fredrikson M. Functional anatomy of hypnotic analgesia: a PET study of patients with fibromyalgia Eur J Pain 1999; 3: 7-12.

72. Oswald LM, Wong DF, Zhou Y et al. Impulsivity and chronic stress are associated with amphetamine-induced striatal dopamine release. Neuroimage. 2007; 36: 153-166.

73. Audenaert K, Peremans K, Goethals I, van Heeringen C. Functional imaging, serotonin and the suicidal brain. Acta Neurol Belg 2006; 106 125-131.

74. Vuilleumier P. Hysterical conversion and brain function. Prog Brain Res 2005; 150: 309-329.

75. De Tiege X, Bier JC, Massat I et al. Regional cerebral glucose metabolism in akinetic catatonia and after remission. J Neurol Neurosurg Psychiatry 2003; 74: 1003-1004.

76. Derry C, Benjamin C, Bladin P et al. Increased serotonin receptor availability in human sleep: evidence from an [18F]MPPF PET study in narcolepsy. Neuroimage 2006; 30: 341-348

77. Mazza S, Soucy JP, Gravel P et al. Assessing whole brain perfusion changes in patients with REM sleep behavior disorder. Neurology 2006; 67: 1618-1622.

78. Smith MT, Perlis ML, Chengazi VU et al. Neuroimaging of NREM sleep in primary insomnia: a Tc-99-HMPAO single photon emission computed tomography study. Sleep 2002; 25: 325-335

79. Joo EY, Hong SB, Tae WS et al. Cerebral perfusion abnormality in narcolepsy with cataplexy. Neuroimage 2005; 28: 410-416.

80. Bassetti C, Vella S, Donati F, Wielepp P, Weder B. SPECT during sleepwalking. Lancet 2000; 356 : 484-485 